\title{
Learning artificial orthographies: Further evidence of a nonanalytic acquisition procedure
}

\author{
BRIAN BYRNE and MARIE CARROLL \\ The University of New England, Armidale, New South Wales, Australia
}

\begin{abstract}
Previous research (Byrne, 1984) showed that adults who learned to read an orthography representing phonetic features (voicing, place of articulation) did not readily obtain usable knowledge of the mapping of phonetic features onto orthographic elements, as evidenced by failure to generalize to partially new stimuli. The present Experiment 1 used a different method of detecting learning savings during acquisition. Subjects learned a set of complex symbols standing for phones, with the elements representing voicing and place. In a second acquisition set, the signs for voicing were reversed. Learning speed was not affected, which was consistent with the claim that feature-element links went unnoticed in initial acquisition. In Experiment 2, some subjects were instructed to "find the rule" embodied in the orthography. None did, and acquisition rates were no different from those of uninstructed subjects. In Experiment 3, subjects had $4 \mathrm{~h}$ of training on the orthography, with consistent feature-symbol mapping for half of the subjects and arbitrary pairings for the remainder. No reaction time advantage emerged in the consistent condition, which is further evidence of nonanalytic acquisition. The results are related to data from children learning to read.
\end{abstract}

One of the present authors recently published a series of experiments (Byrne, 1984) in which adults learned an orthography that represented aspects of the phonetic feature matrix, voicing and place of articulation. That study examined whether, and under what conditions, subjects would gain usable knowledge of the mapping of phonetic structure onto orthographic signs. Such knowledge was detected via a generalization task in which a partially new symbol was presented, one that contained the orthographic sign for, say, +voice. The subject was forced to choose whether the new symbol represented, for example, /f/ or $/ v /$. Above-chance performance was taken to indicate that the subject had learned the speech-symbol relation at the most basic available level: phonetic feature to graphic form. The results indicated that analytic acquisition of that kind occurred only when orthographic training was preceded by separate instruction in elementary articulatory phonetics. The natural tendency was to learn "nonanalytically."

The question most directly at issue - whether and how people can gain usable (i.e., transferable) knowledge of an aspect of linguistic organization over which they already possess tacit perceptual and productive control-is of interest in its own right. This question has been the subject of speculation in the context of access to the "cognitive unconscious"' (Rozin, 1975). The particular vari-

The authors wish to acknowledge the contributions of Doug Tegart and Bob Banens. We also wish to thank David Heap for providing programming assistance. Address correspondence to Brian Byrne, Psychology Department, University of New England, Armidale, New South Wales 2351, Australia. ant of the problem studied in Byrne (1984) has been researched by others (e.g., Healy \& Levitt, 1980). This work has shown that it is not easy for people to "access" phonetic feature structure, even though the contrasts in question are effortlessly incorporated into daily acts of talking and understanding. This is a phenomenon of some interest.

Developments in the study of human learning, however, invite caution about any claim that acquisition has not taken place in some particular instance. Research has shown that experience can leave traces that emerge only with certain measures of learning, and not with others. Of course, it has been acknowledged for a long time that recognition generally produces evidence of learning that does not emerge with recall, but particularly sophisticated demonstrations of the differential sensitivity of measures of learning have more recently been provided by Brooks (1977), Jacoby and Dallas (1981), and Moscovitch, Winocur, and McLachlan (1986). It may be premature to conclude, as Byrne did, "that it was quite difficult for subjects to notice the systematic basis of the orthography" (1984, p. 187). A different measure may, in fact, reveal analytic acquisition.

There is another rather more pragmatic reason for our current attempt to clarify how subjects learn the experimental orthography in question. The original experiments were conceived as an analogy to the task confronting children learning to read an alphabetic script. Children, too, are faced with a system representing the speech stream, where the associations between print and speech could be established at the most basic level available (roughly, letter and phoneme) or at some higher level 
(e.g., printed and spoken word). There are enough parallels between the laboratory and the classroom to keep alive the hope that the experimental task may be an efficient way of pursuing issues in reading acquisition. Parallels include the fact that both adults and children do not initially have conscious access to the relevant language structures (feature and phoneme-for evidence about children, see the considerable literature on phonemic awareness in preliterates, summarized, e.g., in Williams, 1980; for data on adults, see Healy \& Levitt, 1980) and the fact that independent instruction in the relevant linguistic structures assists analytic acquisition of the orthography (for a classroom example, see Williams, 1980). Furthermore, the forced-choice generalization technique developed by Byrne (1984) has subsequently been used with preschoolers (Byrne, in press; Byrne \& Fielding-Barnsley, in press). So far, these studies have revealed a strong tendency toward a nonanalytic acquisition procedure. For example, preliterate children who know how to read only fat and bat fail in forced-choice generalization tasks when required to choose whether fun says "fun" or "bun." Since the adult work is providing a methodological model for the child research, it is important to know whether other learning measures would reveal evidence of analytic learning where no such evidence now exists.

Experiment 1 used a savings paradigm. Two groups of subjects each learned two sets of phoneme-symbol associations. For the experimental group, the signs representing +voice and -voice were reversed between the first and second sets of items without the subjects' being told. For the other subject group, the system remained consistent. If some tacit learning of the mapping between voicing and orthography had taken place during acquisition of the first set, the experimental group should be disrupted in learning the second set.

\section{EXPERIMENT 1}

\section{Method}

Subjects. Twenty-four undergraduate volunteers, tested individually, participated. Twelve were randomly assigned to each of two conditions. All were native speakers of English.

Procedure. Each subject was shown a set of four cards, one at a time. On each card was a symbol (see, e.g., Figure 1, Set 1). The experimenter said aloud the sound of each symbol as the cards were presented twice. First, the sound itself was presented alone, and then the sound as it occurred in a word (e.g., "This is the sound $\varnothing$; this sound occurs in the word 'they." "). The subject then attempted to say the sounds of the symbols when the cards were presented, and the experimenter corrected errors. The criterion for learning was three successive successful passes through the fouritem list. Both errors and number of trials to criterion were recorded.

When the first set had been learned, the experimenter presented a new set of four cards to be learned in the same way. The second set was either consistent or inconsistent with the system from which the first set was derived (see Materials and Design, below). No mention was made of the relation or lack of relation between the first and second sets. Again, errors and trials to criterion were noted.

The consistent subjects were also required to perform the forcedchoice transfer task of Byrne (1984). The transfer items are shown in the lower part of Figure 1. The procedure was identical to that of Experiment 3 in Byrne (1984). Subjects learned eight phoneme-symbol associations and were tested for transfer on the remaining four pairs of English consonants distinguished by voicing. As an example of the transfer task, the subject is presented with the symbol for $/ \mathrm{g} /$ and asked if it represents $/ \mathrm{g} /$ or $/ \mathrm{k} /$. (Transfer data from inconsistent subjects would be uninterpretable since voicing was represented inconsistently throughout the training phase.)

Materials and Design. The materials were the 16 symbol-sound pairs shown in Figure 1. Phonemes that share place and manner of articulation (e.g., /z/ and /s/) have the upper portions of their symbols in common, and shared voicing status (as in, e.g., $/ z /$ and $/ \mathrm{b} /$ ) is indicated by common lower portions. The training stimuli consisted of the 8 training symbols divided into two sets of 4 . Set 1 included $/ \mathrm{d} /, / \mathrm{t} /, / \mathrm{d} /$, and $/ \theta /$; Set 2 comprised $/ \mathrm{b} /, / \mathrm{p} /, / \mathrm{z} /$, and $/ \mathrm{s} /$. In the consistent condition, the relations between symbol and

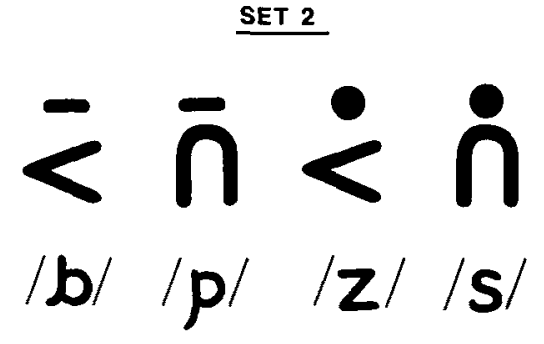

TRANSFER ITEM

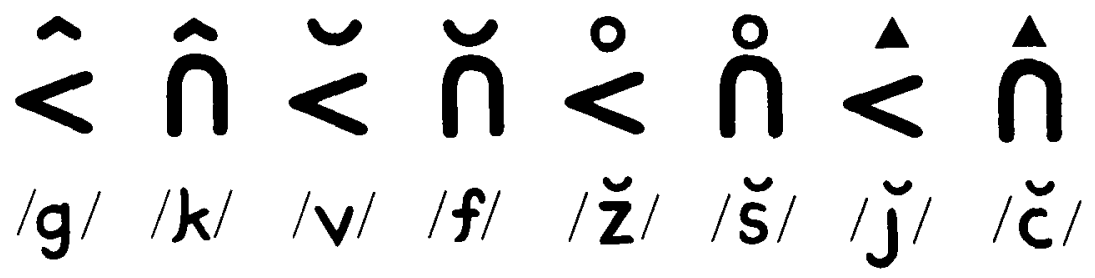

Figure 1. Symbols and corresponding sounds used in Experiments 1 and 2. 
sound for both sets were exactly as they appear in Figure 1. Set 1 or 2 was presented first to half the subjects. In the inconsistent condition, Set 1 or 2 was presented first to half the subjects, and this first-presented set retained the same symbol-sound relationship as shown in Figure 1. However, the second set reversed the relationship between symbol and sound, such that the previously tvoice symbol $(<)$ now represented -voice, and vice versa.

\section{Results and Discussion}

The numbers of errors subjects produced during the acquisition phase are presented in Table 1 . The data are collapsed over sets ( 1 and 2$)$, but we should mention that more errors were made on Set $1(/ \mathrm{d} /, / \mathrm{t} /, / \mathrm{d} /, / \theta /)$ than on Set $2(/ \mathrm{b} /, / \mathrm{p} /, / \mathrm{z} /, / \mathrm{s} /)$; the totals were 131 and 70 , respectively $[t(23)=2.51, p<.05]$. We have no ready explanation for this discrepancy. It was apparently not due to a common spelling pattern, th, for two of the phonemes in Set 1 , because there were no more confusions between this pair than between $/ \mathrm{d} /$ and $/ \mathrm{t} /$.

A 2 (first vs. second set) $\times 2$ (consistent vs. inconsistent) repeated-measures analysis of variance produced no significant main or interaction effects $(F \mathrm{~s} \leq 0.9$, $p s>.51$ ). Thus, the inconsistent subjects were not confused by the abrupt reversal of the voicing symbols halfway through training, nor did the consistent group show savings on the second set of symbols. It is unlikely that this would have been the case if either group of subjects had been learning the orthography analytically, mapping phonetic features onto graphic symbols. The results are thus in line with the general picture that emerged in the research of Byrne (1984).

The consistent subjects' mean transfer score was 4.58 $(S D=1.16)$, not significantly above chance $[t(11)=$ $1.726, p>.05]$. This result replicates Experiment 3 of Byrne (1984), and provides converging evidence for the conclusion that subjects were not learning the basic properties of the experimental orthography.

\section{EXPERIMENT 2}

In this study, we instructed some of the subjects to look for the basic principles of the experimental orthography while learning it and compared these subjects' performance with that of control subjects who had received no such instructions. The literature reports varying outcomes of this sort of manipulation. On the one hand, work by Reber (1976) showed that a "find-the-system"' set slows acquisition of a complex artificial syntax, because, Reber claimed, the subject engages in futile rule search that does not correspond to the complex structure. Also, engaging in explicit rule search jeopardizes the implicit learning process that operates under a neutral set (Reber, 1976; Reber \& Millward, 1968). On the other hand, the rulelearning literature reports that instructions that draw attention to a rule generally accelerate learning (e.g., Jones, 1974). Reber suggested that this variation in outcome is due to structural complexity; where the stimulus pattern is relatively simple, and codable in terms of some wellpracticed heuristic, rule search helps. Where the principles governing experimental items are complex, however, search serves to mislead subjects, producing poor performance.

In the present case, it is not clear what to expect. The "rules" linking phonemes and the orthography are not complex in the ordinary sense; for example, half of each graphic symbol is determined by only one alternation, voicing. They are not simple either, however, in the usual sense of being an extension of a readily retrievable rule system (alternating events, event run length, complementation, etc.). All the data so far suggest that phonetic features are not readily accessed to form the basis of further learning. In a sense, therefore, what our subjects faced was rule discovery, rather than rule identification. As Reber (1976) pointed out, it is the latter, not the former, that is nearly always studied in psychological experimentation. We hypothesized that the difficulty of accessing phonetic features would make it likely that subjects searching for governing principles would be adversely affected during orthography learning in the same way as are subjects searching for a complex rule system.

\section{Method}

Subjects. Twelve undergraduates participated in the implicit instruction group, and another 12 in the explicit instruction group. None had participated in Experiment 1.

Procedure. Each subject was given one of two sets of verbal instructions. The implicit instructions were as follows: "This is a memory experiment. I am going to teach you a new writing system. I will show you a symbol and tell you the sound it represents. After that, you try to make the sound for each letter, and I will tell you if you are right. If you're wrong, I'll tell you what the sound should be. We'll keep going until you can go through all eight symbols correctly three times." The explicit instructions contained the above statement, plus the following: "The symbols are related to the sounds in a systematic way; that is, parts of the symbols stand for aspects of the sounds, so that two sounds that are alike in some way will have symbols that are alike in some way. Since there are

Table 1

Errors and Trials to Criterion in Experiment 1 Training Condition

\begin{tabular}{lcccc} 
& \multicolumn{2}{c}{ Consistent } & \multicolumn{2}{c}{ Inconsistent } \\
\cline { 2 - 5 } \cline { 4 - 5 } & 1st Presented & 2nd Presented & 1st Presented & 2nd Presented \\
\cline { 3 - 5 } Mean Errors & 3.42 & 4.42 & 4.75 & 4.17 \\
$S D$ & 4.98 & 3.78 & 3.79 & 3.51 \\
Mean Trials & & & & \\
to Criterion* & 5.83 & 6.66 & 7.92 & 7.25 \\
$S D$ & 3.54 & 3.23 & 3.99 & 3.39 \\
*Minimum trials to criterion $=3$. & & &
\end{tabular}


rather a lot of new symbols to be learned, it will be to your advantage to try to figure out how the system works. Knowing this will certainly help you in learning and memorizing the items."

The subject was then presented with a set of eight cards, on each of which a symbol was drawn. The training symbols from Experiment $\mathrm{l}$ (Sets 1 and 2) were used. The cards were shown individually in a random order. At the same time, the experimenter said aloud the sound of the symbol. When the eight cards had been presented, the experimenter repeated the procedure. The subject then attempted the sound for each card, and the procedure was repeated until there were three successive successful passes. The experimenter corrected the subject's errors. Both errors and number of trials to criterion were recorded.

At the completion of training, both groups were subjected to the transfer task with the eight unlearned symbols of Figure 1, using the transfer procedure for the consistent group in Experiment 1 and in Byrne (1984). At the end of the generalization, the subjects were questioned about any system they had used to help them during training, and whether they had found this system of use during generalization. Other comments were also noted

\section{Results and Discussion}

Two subjects, one from the explicit and one from the implicit group, failed to reach the learning criterion in less than 23 trials. They were omitted from the analysis, reducing the number in each group to 11 .

The mean number of errors to criterion was $30.5(S D=$ $16.0)$ for the implicit group and $27.8(S D=17.8)$ for the explicit group. This difference was not significant $[t(20)=.36]$. The two groups did not differ in the number of trials to criterion (implicit- $M=14.1, S D=5.44$; explicit $-M=12.7, S D=4.64)[t(20)=.6]$. The large individual differences in learning seen here were observed previously in Byrne (1984).

The groups did not differ in the number of correctly classified symbols in generalization. The mean number correct for the implicit group was $4.27(S D=1.01)$ and for the explicit group was $4.64(S D=1.50)$. Neither of these means was significantly above the chance level of $4[t(10)=.89, p>.05 ; t(10)=1.41, p>.05]$. The ranges for the two groups were also the same: In the implicit group, 2 subjects had scores of 6 out of 8 correct, whereas 2 explicit instruction subjects had scores of 6 and 1 had a score of 7 . A score of 7 out of 8 has a probability of about .03 , so by a .05 criterion, that subject can be said to have fathomed the system.

Subjects' comments during the interrogation revealed that the majority had no idea of a system that might assist them. Some subjects reported relating letter to sound using visual similarity (e.g., " $\cap$ looks like the letter p"), some said they were too busy trying to memorize the sound-symbol relationship to deduce a system, and some did appear to achieve partial insight (e.g., one subject realized that the bottom symbol was particularly important; another said that $\cap$ seemed softer, whereas for symbols containing < "the sound comes a lot quicker"; and another said that < was a hard sound and $\cap$ a soft sound). The comments from subjects performing the generalization task also showed little insight. Some commented on the "softness" of sounds with $\cap$, and one subject noted that some symbols "keep going" while others seem "cut off." Nevertheless, the symbols that this latter subject chose at transfer were incorrect. The explicit subject who correctly classified seven of eight symbols seemed to understand that symbols were in pairs, but could not describe how she differentiated members of a pair. Of the 5 subjects scoring six or more correct in generalization, 3 showed some partial insight into the critical features. No subject, however, mentioned the voiced/ unvoiced distinction, even in nontechnical terminology.

That the explicit instructions did not adversely affect learning casts some doubt on the analogy between discovering the mapping principle and identifying a complex rule system. This doubt may well be justified if it is assumed that phonetic features are in fact very difficult for people to access, as has been our contention. The detrimental effect of search instructions depends on subjects' developing abstractions that are inaccurate representations of the underlying structure, according to Reber's (1976) analysis. Subjects are misled, in other words. However, if they cannot gain a "toehold" on the mapping system because of the very opacity of the components of the sounds in question, they cannot be misled. This would also readily explain why the explicit group did not perform better than the implicit group in acquiring what, after all, is a structurally very simple system.

\section{EXPERIMENT 3}

The results of Experiments 1 and 2 were essentially null results: no savings and no effects of search instructions. There are several well-recognized difficulties with null findings, and it is proper that we should try to address them. One possibility is that the learning conditions did not put sufficient pressure on the subjects to force analytic learning. There were only eight associations to be formed, and rote learning may have been sufficient. Two results from Byrne's (1984) work made us doubt that simple rote learning always operates when the number of items is eight or less: subjects did acquire the rule system with only four items when given prior instruction in articulatory phonetics (Experiment 5) and when the symbols were mapped onto the more transparent phonological components of syllable onset and rime (Experiment 2). With opaque sound structures, however, analytic learning may emerge only when a more demanding experimental regime is imposed.

A second difficulty with null results concerns statistical power. In Experiments 1 and 2, subject numbers were 11 or 12 per condition, not unusually small for cognition experiments, but not large either, especially if weak effects may be involved. In defense, we point to the earlier experiments of Byrne (1984), in which three other groups of subjects of about the same size exhibited similar null effects under a variety of conditions. In all, therefore, a sizable number of literate and intelligent people have failed to detect feature-symbol mappings after learning the ex- 
perimental orthography. Nevertheless, another study using extensive exposure to the system would be useful against the null result background.

We based Experiment 3 on an experiment by Brooks (1977). He created new, nonalphabetic symbols for a group of six words, and timed subjects as they repeatedly read through the list-a total of 2,400 responses in 400 trials. For half the subjects the new symbols were related systematically to the phonemes in the words, and for the remaining subjects the pairings between printed and spoken words were arbitrary. Brooks found that the systematic subjects achieved lower list-reading times over about the last 200 trials, showing that they could take advantage of the systematic orthography.

In our Experiment 3, half the subjects learned the symbol-phoneme pairings of Experiment 1 (consistent condition). The remainder learned arbitrary pairings of symbols and phonemes. In Brooks's (1977) study, subjects were given prior training in symbol-sound pairings prior to learning to read the systematic test. In our experiment, this prior training was omitted, because we already knew from Byrne's (1984, Experiments 5 and 6) research that teaching subjects how the symbols represent phonetic features immediately results in analytic acquisition. Instead, we addressed the question of whether learning to use an orthography that represents phonemes would be more rapid if the symbols were systematically related to phonetic features than if they were not. Brooks's data are relevant in that they show that if subjects do know the mapping of symbol onto sound, then response latency is lower than if they do not (because they cannot). We used reaction time (RT) as a way of discerning whether subjects induce the organizing principle of the orthography through extended exposure.

\section{Method}

Subjects. Sixteen undergraduate students and departmental staff, none of whom had participated in earlier experiments, were recruited for this study and paid $\$ 20$ for their four hour-long sessions. All were native English speakers

Procedure. The subjects sat facing a computer-driven display that showed the experimental orthographic symbols, along with the standard English letters representing the associated phonemes. The symbol-letter pairs were arranged in two rows of four on the top half of the video display unit. The eight stimuli for this experiment were the symbols representing the following phonemes in Figure 1: $/ \mathrm{b} /, / \mathrm{p} /, / \mathrm{v} /, / \mathrm{f} /, / \mathrm{t} /, / \mathrm{d} /, / \mathrm{s} /$, and $/ \mathrm{z} /$. For 8 subjects, the symbols were systematically related to the phonetic features, and for the other 8 , they were nonsystematic, as outlined earlier. We refer to the two conditions as systematic and nonsystematic. On each trial, a single experimental symbol appeared in a box under the fixed display, and the subjects were instructed to say its sound as quickly as possible. In the case of the stop phonemes $(/ \mathrm{b} /, / \mathrm{p} /, / \mathrm{d} /, / \mathrm{t} /)$, the consonant was pronounced with /a/ following. The subjects' responses were timed from stimulus onset via a voice-operated relay and stored on computer. An experimenter who could also see the display recorded errors. Within a trial block, the eight items were each presented once in an otherwise random order. Rests were available to the subjects between trial blocks, under their own con- trol. In a single session, 75 blocks were presented in the space of about $1 \mathrm{~h}$. Each subject participated in four such sessions, one per day, on consecutive days

\section{Results and Discussion}

As might be expected, error rates were very low (less than .004), because the subjects could view the symbols and their associated letters throughout the experiment. Error trials were included in computing response times.

We averaged each subject's responses over each block of 15 trials, generating 5 points per day and 20 points for the entire experiment. The means of these data points across subjects within treatment groups are presented in Figure 2. There was a clear trials effect $[F(1,19)=22.03$, $p<.001]$, but no hint of group or interaction effects $[F(1,14)=0.02$ for group, and $F(1,19)=0.88$ for the interaction] (see Figure 2 ). The subjects clearly responded more quickly as a function of practice, but no advantage accrued to the subjects for whom the orthography systematically represented phonetic features. Their failure to capitalize on the systematic nature of the orthography confirms the relative opacity of phonetic features. Even after 2,400 exposures to exemplars of an orthography representing a small stock of phonetic features, including the only two values of voicing employed by English, no trace of the system appeared in response latency.

\section{GENERAL DISCUSSION}

Byrne (1984) showed that adults could not easily learn the mapping of orthographic elements onto phonetic elements in a feature-based orthography. The acquisition procedure was thus nonanalytic, similar to that of children who can read cat without any knowledge of what the individual letters represent. It is possible, however, that there may have been acquisition of the underlying rule below a level that the transfer test may detect, and even in the presence of explicit denials that there was anything to learn (see Brooks, 1977, 1978, in press; Reber, 1967). A different technique might expose evidence of subjects' having acquired the orthographic principle. This was the rationale for the savings experiment (Experiment 1). Would there be implicit learning? The outcome of that experiment was clear: The artificial orthography was acquired in nonanalytic fashion, and there was no demonstrable savings in learning a consistent orthography over an inconsistent orthography.

In Experiment 2 we examined the effect of an instruction to find the rule linking symbols and phonetic features on acquisition rates. This instruction has been shown to slow learning speed when the rule is complex (Reber, 1976), but to accelerate it when the rule is relatively simple (Jones, 1974). We found no effect of the find-the-rule instruction, a result that we interpreted as confirming the difficulty of accessing phonetic feature structure. Even though the orthography is related to the components of 


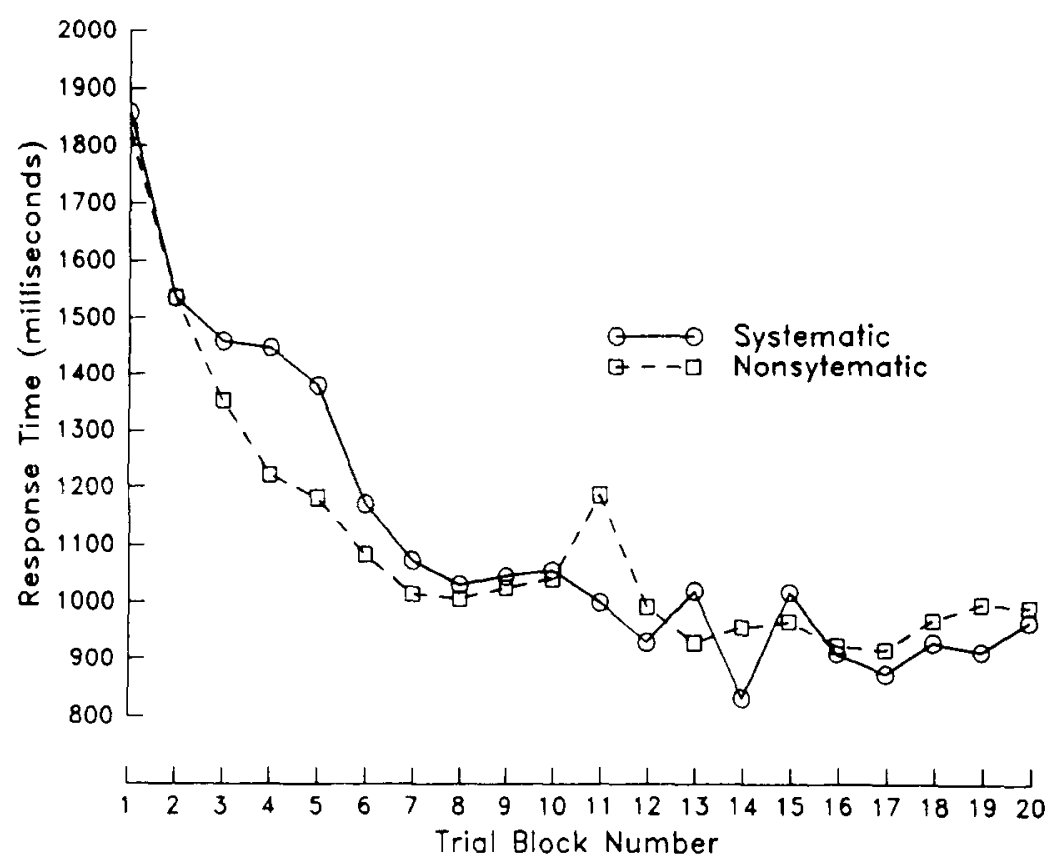

Figure 2. Average scores for blocks of 15 trials in Experiment 3.

the phonemes in a structurally simple way, learning is not assisted because the subjects cannot penetrate the phonetic feature matrix. However, they are not misled by false hypotheses about the linking rule for exactly the same reason: they cannot begin to form such hypotheses.

Experiment 3 offered subjects extended exposure to the orthography under systematic and arbitrary mappings, but found no evidence of speedier performance under the former condition. These results using the dependent variable of reaction time converge with earlier data in demonstrating nonanalytic acquisition.

Studies reported in Byrne (in press) and in Byrne and Fielding-Barnsley (in press) indicate that the same nonanalytic learning procedure dominates the very earliest stages of the child's acquisition of reading, and investigations by Seymour and Elder (1986) demonstrate that the tendency is a persistent one. Those researchers followed the reading progress of beginning readers for a year while they were being taught by a fairly strict "wholeword" method. The children were largely incapable of reading previously unseen words throughout the period of the study. The training techniques we used in these experiments parallel those of the whole-word method, in that independent instruction in the basic correspondences of the orthography is not given in either case. It appears that the assumption that learners will automatically fathom the system's organizations is flawed in both cases. Rather, nonanalytic acquisition of orthographies that map segments as small as or smaller than the phoneme may be characteristic of the early stages of learning. What requires explanation, therefore, is how the grip of this tendency is finally broken, in both children and adults. Byrne's
(1984) studies suggest one point of leverage: provision of independent information about the speech structures reflected in the orthography. This finding is nicely mirrored by current suggestions (e.g., Bradley \& Bryant, 1983; Juel, Griffith, \& Gough, 1986) that as children learn to read an alphabetic script, they can be assisted by instruction in the phonemic organization of speech.

\section{REFERENCES}

Bradley, L., \& Bryant, P. E. (1983). Categorizing sounds and leaming to read: A causal connection. Nature, 301, 419-421.

Brooks, L. R. (1977). Visual pattern in fluent word identification. In A. S. Reber \& D. L. Scarborough (Eds.), Toward a psychology of reading: The proceedings of the CUNY conference (pp. 143-181). Hillsdale, NJ: Erlbaum.

Brooks, L. R. (1978). Nonanalytic concept formation and memory for instances. In E. Rosch \& B. B. Lloyd (Eds.), Cognition and categorization (pp. 169-211). Hillsdale, NJ: Erlbaum.

Brooks, L. R. (in press). Decentralized control of categorization: The role of prior processing episodes. In U. Neisser (Ed.), Categories reconsidered: The ecological and intellectual bases of categories. Cambridge: Cambridge University Press.

BYRNE, B. (1984). On teaching articulatory phonetics via an orthography. Memory \& Cognition, 12, 181-189.

BYRNE, B. (in press). Studies in the unbiased acquisition procedure for reading: Rationale, hypotheses, and data. In P. B. Gough (Ed.), Reading acquisition. Hillsdale, NJ: Erlbaum.

Byrne, B., Fielding-Barnsley, R. (in press). Phonemic awareness and letter knowledge in the child's acquisition of the alphabetic principle. Journal of Educational Psychology.

Healy, A. F., Levit, A. G. (1980). Accessibility of the voicing distinction for learning phonological rules. Memory \& Cognition, 8, 107-114.

JACOBY, L. L., DALLAS, M. (1981). On the relationship between autobiographical memory and perceptual learning. Joumal of Experimental Psychology: General, 110, 306-340. 
Jones, M. R. (1974). Cognitive representations of serial patterns. In B. H. Kantowitz (Ed.), Human information processing: Tutorials in performance and cognition (pp. 187-229). Potomac, MD: Erlbaum.

Juel, C., Griffith, P. L., \& Gough, P. B. (1986). Acquisition of literacy: A longitudinal study of children in first and second grade. Journal of Educational Psychology, 78, 243-255.

Moscovitch, M., Winocur, G., \& McLachlan, D. (1986). Memory as assessed by recognition and reading in normal and memory-impaired people with Alzheimer's disease and other neurological disorders. Journal of Experimental Psychology: General, 115, 331-347.

REBER, A. S. (1967). Implicit learning of artificial grammars. Journal of Verbal Learning \& Verbal Behavior, 6, 855-863.

REBer, A. S. (1976). Implicit learning of synthetic languages: The role of instructional set. Journal of Experimental Psychology: Human Learning \& Memory, 2, 88-94.
Reber, A. S., Millward, R. B. (1968). Event observation in probability learning. Joumal of Experimental Psychology, 77, 317-327. Rozin, P. (1975). The evolution of intelligence and access to the cognitive unconscious. In J. M. Sprague \& A. N. Epstein (Eds.), Progress in psychobiology and physiological psychology (Vol. 6, pp. 245-280). New York: Academic Press.

Seymour, P. H. K., Elder, L. (1986). Beginning reading without phonology. Cognitive Neuropsychology, 3, 1-36.

WiLliams, J. (1980). Teaching decoding with an emphasis on phoneme analysis and phoneme blending. Journal of Educational Psychology, $72,1-15$.

(Manuscript received April 17, 1987; revision accepted for publication August 30, 1988.) 